\title{
Artículo Original \\ Impacto de la Pandemia por COVID-19 sobre la Atención del Infarto de Miocardio ST Elevado en el Perú
}

Piero Custodio-Sánchez, ${ }^{1 *}$ David Miranda, ${ }^{2}$ Luis Murillo ${ }^{3}$

Recibido 14 de abril de 2020 Aceptado 25 de mayo de 2020

Afiliaciones de los Autores: ${ }^{1}$ Servicio de Cardiología. Unidad de Cardiología Intervencionista. Hospital Nacional Almanzor Aguinaga Asenjo Essalud, Chiclayo, Perú.

${ }^{2}$ Servicio de Cardiología Clínica. Instituto Nacional Cardiovascular INCOR. Lima, Perú.

${ }^{3}$ Médico residente de Cardiología. Instituto Nacional Cardiovascular INCOR. Lima, Perú.

\section{*Correspondencia:} Hospital Nacional Almanzor Aguinaga Asenjo Essalud. Plaza De La Seguridad Social S/N. Chiclayo. Perú.

Telef. 074-481120, anexo 3453.

\section{Correo:}

custodiomed@hotmail.com

\section{Conflicto de interés:}

No se reporta conflicto de interés.

\section{Responsabilidades éticas:}

Protección de personas y animales.

Los autores declaran que para esta investigación no se ha realizado experimentos en seres humanos $\mathrm{ni}$ en animales.

Confidencialidad de los datos: Los autores declaran que han seguido los protocolos de su centro de trabajo sobre la publicación de datos de pacientes.

\section{Derecho a la privacidad y} consentimiento informado: Los autores declaran que en este artículo no aparecen datos de pacientes.

\section{Financiación}

Ninguna.
Objetivo: Comparar el número de ingresos, características clínicas y resultados terapéuticos de los pacientes atendidos por infarto de miocardio con elevación del segmento ST (IMCEST) antes y durante el estado de emergencia por COVID-19 en Perú.

Métodos: Se realizó un estudio observacional analítico de cohortes retrospectivas, derivadas del PEruvian Registry of ST-segment Elevation Myocardial Infarction II (PERSTEMI II). Se comparó los pacientes atendidos por IMCEST 45 días antes y durante los 45 días iniciales del estado de emergencia por COVID-19 en Perú.

Resultados: Durante los primeros 45 días del período de emergencia se encontró una disminución del $59 \%$ en el número de ingresos por IMCEST. Hubo una mayor proporción de pacientes con antecedentes de hipertensión arterial y dislipidemia. Se observó una tendencia a reducción en el acceso a terapias de reperfusión (73.5\% vs $66.6 \%)$, siendo la fibrinólisis la terapia más utilizada. El motivo más frecuente de no reperfusión fue la presentación tardía $>24$ horas $(41.7 \%, p=0.004)$. Hubo una tendencia a reducción del tiempo al primer contacto médico y del tiempo de isquemia hasta la reperfusión. Se registró menor incidencia de falla cardíaca post infarto. La mortalidad fue similar en ambos grupos (3.4\% vs $2.7 \%)$.

Conclusiones: La pandemia por COVID-19 en el Perú ha generado una disminución significativa en los ingresos por IMCEST, además de una tendencia a menor empleo de terapias de reperfusión. La presentación tardía de los pacientes fue la causa más frecuente de no reperfusión.

Palabras clave: infarto de miocardio • COVID-19 • SARS-CoV2.

\section{Impact of the COVID-19 pandemic on}

ABSTRACT

\section{ST- elevation myocardial infarction care in Peru}

Purpose: To compare the number of admissions, clinical features and therapeutic outcomes of patients treated for acute ST-segment elevation myocardial infarction (STEMI), before and after the COVID-19 pandemic state of emergency in Peru.

Methods: Observational analytical study of retrospective cohorts, derivated from the PEruvian Registry of ST-segment Elevation Myocardial Infarction II (PERSTEMI II). We compared the patients treated for STEMI, between 45 days before and during the first 45 days of the COVID-19 state of emergency in Peru.

Results: During the first 45 days of the COVID-19 state of emergency, the team found a $59 \%$ decrease on the number of admissions for STEMI. There was a larger proportion of patients with high blood pressure and dyslipidemia. We noticed a decreasing trend in the access to reperfusion therapies $73 \%$ vs. $66.6 \%)$; the fibrinolysis was the most commonly used therapy. The most frequent reason for no reperfusion was the late onset $>24$ hours $(41.7 \%, p=0.004)$. There was a trend of time reduction to first medical contact and less ischemia time to reperfusion. A lower incidence of post-infarction heart failure was registered. The mortality was similar in both groups (3.4\% vs. $2.7 \%)$.

Conclusions: COVID-19 pandemic in Peru has generated a significant reduction of STEMI admissions and a trend in less use of reperfusion therapies. The late onset of patients was the most common reason of not reperfusion.

Key words: myocardial infarction • COVID-19 • SARS-CoV2. 
$\mathrm{E}$ $\mathrm{n}$ las dos últimas décadas, el mundo ha enfrentado tres emergencias de salud pública provocadas por la familia de Coronavirus. En el año 2002, el Síndrome respiratorio agudo severo (SARS-CoV) generó 8422 infecciones y 916 muertes. Posteriormente, en el año 2012, el Síndrome respiratorio del oriente medio (MERS-CoV) generó 1600 infecciones y 574 muertes.' Recientemente, en diciembre del 2019, en China, se reportó el primer caso de enfermedad por el Nuevo Coronavirus 2019 (COVID - 19) producida por el SARS-CoV2; originando hasta finales de abril del 2020, 3 millones de personas contagiadas y más de 200000 muertes, siendo considerada en la actualidad una pandemia. ${ }^{2-3} \mathrm{~A}$ nivel nacional, hasta finales de abril del 2020, se reportaron alrededor de 34,000 pacientes diagnosticados $y$ más de 1000 muertes. Debido a la rápida propagación de la infección se realizó una reorganización en el sistema de salud, y se declaró el estado de emergencia con aislamiento social obligatorio en el Perú desde el 16 de marzo del $2020 .{ }^{4}$ El resultado de este nuevo escenario ha afectado la atención de las enfermedades cardiovasculares, siendo una de ellas el infarto de miocardio con elevación del segmento ST (IMCEST). ${ }^{5}$

EI IMCEST es una de las causas más frecuentes de enfermedad cardiovascular en la población general. ${ }^{6}$ En el Perú se reporta una mortalidad intrahospitalaria de $10.1 \%,{ }^{7}$ la cual está influida por múltiples factores como la edad, comorbilidades y demora en la administración del tratamiento. ${ }^{8}$ En los últimos meses, producto de la pandemia por COVID-19, se ha observado a nivel mundial una clara disminución del número de pacientes ingresados a emergencias con el diagnóstico de IMCEST y de las intervenciones coronarias percutáneas para su tratamiento. $^{9-10}$

En el Perú, no se conoce el impacto que la pandemia ha originado en la atención del IMCEST. Por ello, el objetivo del presente estudio es comparar el número de ingresos, características clínicas y resultados terapéuticos de los pacientes atendidos por IMCEST 45 días antes y durante los primeros 45 días del estado de emergencia por COVID-19 en el Perú.

\section{Material y Método}

Realizamos un estudio observacional analítico de cohortes retrospectivas, derivadas del actual registro nacional de IMCEST: "Peruvian Registry of ST elevation myocardial infarction - II" (PERSTEMI II) del 2020; el cual, es una iniciativa para conocer la realidad del tratamiento del IMCEST en el Perú. Este viene registrando vía electrónica, todos los casos ingresados en los hospitales públicos, de las fuerzas armadas y clínicas privadas, de las principales capitales de departamento del Perú, que aceptaron participar en el estudio; inició en enero 2020 y tiene planificado terminar en diciembre 2020. Criterios de inclusión: pacientes mayores de 18 años, de ambos sexos, con síntomas isquémicos o equivalentes, con elevación persistente del segmento ST $>0.1 \mathrm{mV}$ (o > $0.2 \mathrm{mV}$ en V1 y V2) en 2 o más derivaciones contiguas, independientemente del tiempo de evolución, o con bloqueo completo de rama izquierda del haz de His, presumiblemente de novo. Criterios de exclusión: pacientes con síndrome coronario sin elevación del segmento ST, o embolismo coronario.

Para el presente subanálisis la primera cohorte incluyó pacientes con IMCEST diagnosticados antes de la declaratoria del estado de emergencia por el COVID 19 (dada el 16 de marzo del 2020) y se comparó con la cohorte de pacientes con IMCEST tratados luego de la declaratoria en pleno estado de emergencia. Debido a que el tiempo de cuarentena transcurrido hasta el punto de corte para este estudio fue de 45 días, se decidió comparar a los pacientes registrados en el estudio PERSTEMI II desde 45 días antes, hasta 45 días después del 16 de marzo. Por tanto, la primera cohorte comprendió los casos atendidos del 01 de febrero al 15 de marzo del 2020 y la segunda cohorte del 16 de marzo al 30 de abril del 2020.

No se realizó la comparación con pacientes atendidos con IMCEST en los meses de marzo y abril del 2019, porque no se tienen esos datos, ya que el registro PERSTEMI II fue iniciado recién en enero del 2020.

Se evaluaron las características generales de la población (edad, sexo, lugar de atención), comparando los tiempos de isquemia, de primer contacto médico, tipo de reperfusión, porcentaje de pacientes a los que se les aplica algún tratamiento de reperfusión, porcentaje de no reperfundidos y complicaciones intrahospitalarias que incluyen mortalidad, complicación mecánica y falla cardíaca, entre ambas cohortes.

Los resultados se expresan en frecuencias y porcentajes para variables cualitativas y en medias o medianas con desviación estándar o rango intercuartil en las variables cuantitativas dependiendo de su distribución normal, la cual fue evaluada por el test de normalidad de Shapiro Wilk.

Para el análisis estadístico se usó los test de Chicuadrado para variables cualitativas, T-student para variables cuantitativas de distribución normal y suma de rangos de Wilcoxon para variables cuantitativas de distribución no paramétrica. En todo caso se consideró un valor de $p<0.05$ como de significancia estadística para la diferencia de dos valores. Toda la evaluación estadística se realizó con el programa STATA versión 14.0. 


\section{Resultados}

Entre el 01 de febrero y el 30 de abril del 2020, se registraron 123 casos de IMCEST en el registro PERSTEMI II, atendidos en 9 centros de la ciudad de Lima y 6 centros del interior del país. Durante el estado de emergencia por COVID-19 (período post COVID), se evidenció una importante disminución del número de casos de IMCEST reportados en relación al período previo, lo que constituye una disminución del 59\%. (Figura 1)

Dentro de las características clínicas basales no se encontraron diferencias significativas según la edad, sexo, presentación clínica y electrocardiográfica entre ambas cohortes; sin embargo, durante el estado de emergencia, encontramos una proporción significativamente mayor de pacientes con antecedente de hipertensión arterial y dislipidemia. (Tabla 1) No se reportaron casos de IMCEST en pacientes COVID - 19 positivos durante el tiempo de estudio.

La mayoría de los pacientes recibieron alguna terapia de reperfusión (fibrinólisis o angioplastia primaria); sin embargo, se observó un menor acceso a ellas durante el estado de emergencia, (Figura 2) así como una mayor proporción de fibrinólisis y reducción de la angioplastia primaria. (Tabla 2) La presentación tardía (> 24 horas) fue el motivo más frecuente de falta de administración de alguna terapia de reperfusión durante la pandemia. (Tabla 2) Durante el estado de emergencia hubo menor tiempo al primer contacto médico, menor tiempo de isquemia hasta el uso de alguna terapia de reperfusión y un menor tiempo de isquemia hasta la intervención coronaria percutánea primaria, aunque esto no fue estadísticamente significativo. (Tabla 3) La terapia hospitalaria recibida en ambas etapas no presentó diferencias importantes. La mortalidad intrahospitalaria fue similar en ambas cohortes y se encontró menor proporción de falla cardíaca post infarto durante el estado de emergencia. (Tabla 4)

\section{Discusión}

El presente subanálisis del registro PERSTEMI II muestra una reducción del $59 \%$ en los ingresos por IMCEST durante el estado de emergencia por COVID-19 en el Perú. Este hallazgo es llamativo, ya que existe evidencia de factores que pueden incrementar la incidencia de infarto de miocardio durante infecciones respiratorias agudas. Kwong et al. ${ }^{11}$ reportaron la asociación significativa entre el infarto de miocardio y la infección por influenza durante los primeros 7 días de la misma. Igualmente, durante la infección por coronavirus responsable del SARS aparecido en China el año 2002, se

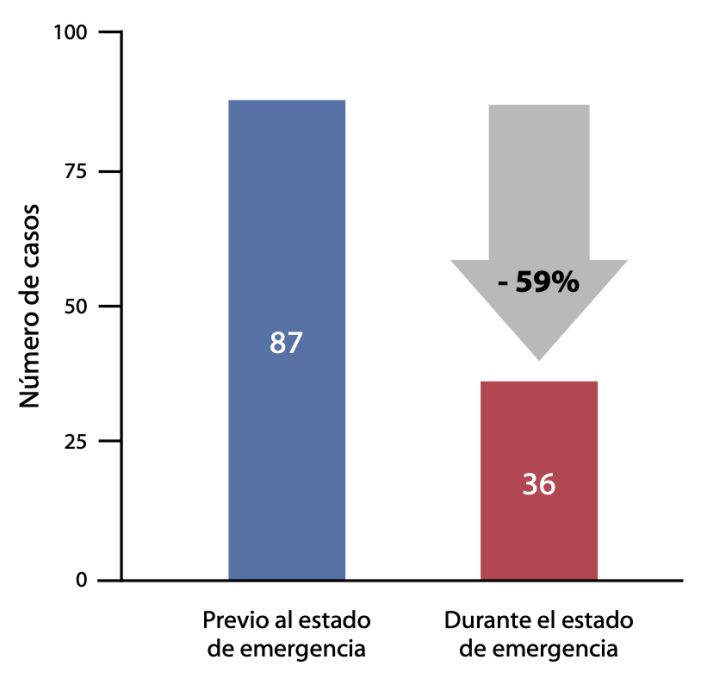

Figura 1. Variación en el número de casos de infarto de miocardio ST elevado, durante la pandemia por COVID 19 en el Perú.

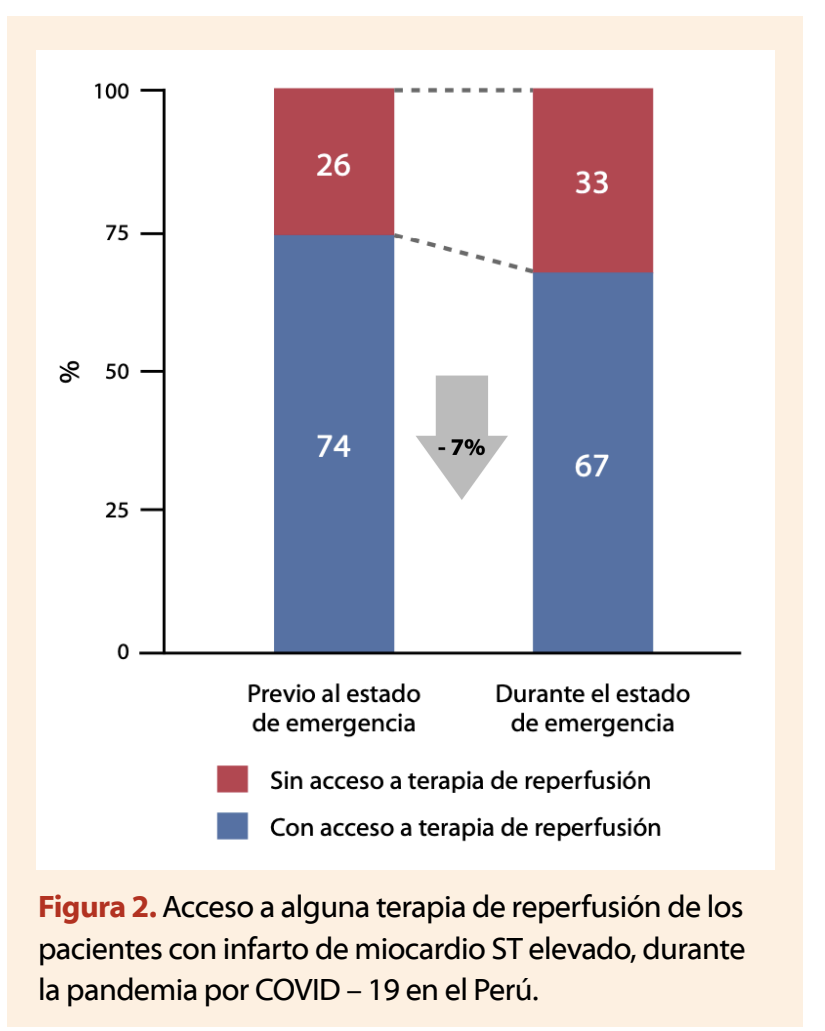

reportaron casos de infarto de miocardio en relación a la inflamación sistémica aguda que generaba el virus. ${ }^{12-13}$

Asimismo, existen factores psicosociales que pueden desarrollarse durante el período de cuarentena, como el estrés agudo relacionado por temor al contagio de la infección o los trastornos de ansiedad y depresión propios del aislamiento, los cuales pueden comportarse como gatillos de infarto de miocardio. ${ }^{14-15}$ Adicionalmente, la cuarentena puede condicionar la falta de adherencia de los pacientes a su 
Tabla 1. Características de los pacientes con IMCEST antes y durante el estado de emergencia por COVID - 19

\begin{tabular}{|c|c|c|c|}
\hline & $\begin{array}{l}\text { Previo al estado } \\
\text { de emergencia } \\
\qquad(\mathbf{n}=\mathbf{8 7})\end{array}$ & $\begin{array}{l}\text { Durante el estado } \\
\text { de emergencia } \\
\qquad(n=36)\end{array}$ & $\mathbf{p}$ \\
\hline Edad promedio & $67( \pm 10)$ & $64( \pm 13)$ & 0.245 \\
\hline Sexo masculino & $73(83.9)$ & 33 (91.6) & 0.257 \\
\hline \multicolumn{4}{|c|}{ Antecedentes y Factores de Riesgo } \\
\hline Hipertensión arterial & $39(44.8)$ & $25(69.4)$ & 0.017 \\
\hline Diabetes mellitus 2 & $30(34.4)$ & $8(22.2)$ & 0.181 \\
\hline Dislipidemia & $39(44.8)$ & $25(69.4)$ & 0.017 \\
\hline Hiperuricemia & $5(3.4)$ & $3(8.3)$ & 0.252 \\
\hline Tabaquismo & $23(26.4)$ & $9(25)$ & 0.869 \\
\hline Enfermedad coronaria estable & $6(6.9)$ & $4(11.1)$ & 0.436 \\
\hline Enfermedad cerebrovascular & $5(5.7)$ & $3(8.3)$ & 0.597 \\
\hline Infarto de miocardio previo & $9(10.3)$ & $4(11.1)$ & 0.9 \\
\hline Enfermedad renal & $5(5.7)$ & $2(5.5)$ & 0.967 \\
\hline Falla cardíaca crónica & $1(1.2)$ & $1(2.7)$ & 0.516 \\
\hline Ritmo por electrocardiograma & & & 0.13 \\
\hline Sinusal & $83(95.4)$ & $35(97.2)$ & \\
\hline Fibrilación auricular & $0(0)$ & $1(2.7)$ & \\
\hline Bloqueo AV de alto grado & $4(4.6)$ & $0(0)$ & \\
\hline Localización del infarto & & & 0.73 \\
\hline Anterior & $12(13.9)$ & $2(5.5)$ & \\
\hline Anteroseptal & $19(22.1)$ & $6(16.7)$ & \\
\hline Anterolateral & $4(4.6)$ & $3(8.3)$ & \\
\hline Anterior extenso & $15(17.4)$ & $6(16.7)$ & \\
\hline Inferior & $23(26.4)$ & $10(27.8)$ & \\
\hline Lateral & $3(3.5)$ & $2(5.5)$ & \\
\hline Inferoposterior & $11(12.8)$ & 7 (19.4) & \\
\hline \multicolumn{4}{|l|}{ Presentación clínica } \\
\hline Dolor anginoso & $84(96.5)$ & $34(94.4)$ & 0.59 \\
\hline Dolor atípico & $2(2.3)$ & $2(5.5)$ & 0.354 \\
\hline Disnea & $30(34.4)$ & $11(30.5)$ & 0.674 \\
\hline Síncope & $2(2.3)$ & $0(0)$ & 0.359 \\
\hline Arresto cardíaco & $1(1.1)$ & $0(0)$ & \\
\hline Killip-Kimball & & & 0.09 \\
\hline 1 & 62 (71.3) & 30 (83.3) & \\
\hline II & $23(26.4)$ & $4(11.1)$ & \\
\hline III & $1(1.1)$ & $0(0)$ & \\
\hline IV & $1(1.1)$ & $2(5.6)$ & \\
\hline Traslado hacia otro hospital & $62(71.3)$ & $23(63.9)$ & 0.421 \\
\hline
\end{tabular}

Se reporta medias ( \pm desviación estándar) y frecuencias (porcentaje) para variables cuantitativas y categóricas, respectivamente. IMCEST: infarto de miocardio con elevación del segmento ST; AV: auriculoventricular. 
Tabla 2. Terapias de reperfusión administradas a los pacientes del estudio

\begin{tabular}{|c|c|c|c|}
\hline & $\begin{array}{l}\text { Previo al estado } \\
\text { de emergencia } \\
\qquad(\mathbf{n}=\mathbf{8 7})\end{array}$ & $\begin{array}{l}\text { Durante el estado } \\
\text { de emergencia } \\
\qquad(n=36)\end{array}$ & $\mathbf{p}$ \\
\hline Alguna terapia de reperfusión & $64(73.5)$ & $24(66.7)$ & 0.441 \\
\hline ICP primaria & $36(41.4)$ & $11(30.6)$ & 0.261 \\
\hline Flbrinólisis & $28(32.1)$ & $13(36.1)$ & 0.612 \\
\hline Éxito de la fibrinólisis & $17(60.7)$ & $10(76.9)$ & 0.308 \\
\hline ICP de rescate & $7(8.0)$ & $2(5.6)$ & 0.629 \\
\hline Estrategia farmacoinvasiva & $13(14.9)$ & $7(19.4)$ & 0.538 \\
\hline Total de coronariografías e ICP & $56(64.4)$ & $20(55.5)$ & 0.360 \\
\hline Flujo TIMI 3 final post ICP & $43(76.8)$ & $18(90)$ & 0.670 \\
\hline Enfermedad multivaso & $27(48.2)$ & $13(65.0)$ & 0.630 \\
\hline ICP de la ANRI & $20(74.1)$ & $5(45.4)$ & 0.092 \\
\hline Ninguna terapia de reperfusión & $23(26.4)$ & $12(33.3)$ & 0.441 \\
\hline Contraindicación a fibrinólisis & $0(0)$ & $0(0)$ & \\
\hline Negación del paciente & $1(4.3)$ & $1(8.3)$ & 0.679 \\
\hline Falta de fibrinolíticos & $5(21.7)$ & $0(0)$ & 0.062 \\
\hline No disponibilidad de ICP & $6(26.1)$ & $0(0)$ & 0.030 \\
\hline Presentación tardía $>12$ horas & $2(8.7)$ & $2(16.7)$ & 0.720 \\
\hline Presentación tardía > 24 horas & $0(0)$ & $5(41.7)$ & 0.004 \\
\hline Presentación tardía > 72 horas & $6(26.1)$ & $1(8.3)$ & 0.102 \\
\hline Otros & $5(21.7)$ & $2(16.7)$ & 0.339 \\
\hline Desconocido & $2(8.7)$ & $0(0)$ & \\
\hline
\end{tabular}

Se reporta frecuencia (porcentaje) para cada variable.

IMCEST: infarto de miocardio con elevación del segmento ST; ICP: intervención coronaria percutánea;

ANRI: arteria no responsable del infarto.

Tabla 3. Componentes del tiempo de isquemia en los pacientes de estudio

\begin{tabular}{|c|c|c|c|}
\hline & $\begin{array}{l}\text { Previo al estado } \\
\text { de emergencia } \\
\qquad(n=87)\end{array}$ & $\begin{array}{c}\text { Durante el estado } \\
\text { de emergencia } \\
(n=36)\end{array}$ & $\mathbf{p}$ \\
\hline Tiempo hasta el primer contacto médico (minutos) & $120(60-240)$ & $90(45-180)$ & 0.281 \\
\hline $\begin{array}{l}\text { Tiempo de isquemia hasta la aplicación de alguna } \\
\text { terapia de reperfusión (minutos) }\end{array}$ & $330(240-1260)$ & $237(150-480)$ & 0.086 \\
\hline Tiempo de isquemia hasta ICP primaria (minutos) & $690(330-1500)$ & $480(225-1200)$ & 0.243 \\
\hline Tiempo puerta - balón (minutos) & $90(50-180)$ & $60(45-100)$ & 0.634 \\
\hline
\end{tabular}

Se reporta mediana (rango intercuartil) para cada variable.

ICP: intervención coronaria percutánea 
Tabla 4. Terapias utilizadas y eventos intrahospitalarios en los pacientes de estudio

\begin{tabular}{lccc}
\hline & $\begin{array}{c}\text { Previo al estado } \\
\text { de emergencia } \\
\mathbf{( n = 8 7 )}\end{array}$ & $\begin{array}{c}\text { Durante el estado } \\
\text { de emergencia } \\
\text { (n= 36) }\end{array}$ & p \\
\hline Terapia hospitalaria & $11(12.6)$ & $2(5.5)$ & 0.245 \\
\hline Inotrópicos & $10(11.5)$ & $2(5.5)$ & 0.313 \\
\hline Vasopresores & $4(4.6)$ & $0(0)$ & 0.191 \\
Balón de contrapulsación intraaórtico & $11(12.6)$ & $2(5.5)$ & 0.245 \\
\hline Ventilación mecánica & & & 0.849 \\
\hline Eventos intrahospitalarios & $3(3.4)$ & $1(2.7)$ & 0.359 \\
\hline Muerte cardiovascular & $2(2.3)$ & $0(0)$ & 0.876 \\
\hline Muerte no cardiovascular & $2(2.3)$ & $1(2.7)$ & 0.958 \\
\hline Complicaciones mecánicas & $7(8.1)$ & $3(8.3)$ & $\mathbf{0 . 0 4 8}$ \\
\hline Shock cardiogénico & $21(24.1)$ & $3(8.3)$ & 0.518 \\
\hline Falla cardíaca & $1(1.1)$ & $0(0)$ & \\
\hline Stroke & & & \\
\hline Se reporta frecuencia (porcentaje) para cada variable. & & & \\
\hline
\end{tabular}

terapia habitual ante la dificultad para conseguir medicación por restricciones de movimiento o el temor al contagio de la infección al acudir a hospitales, lo que lleva a la pérdida de control de los factores de riesgo tradicionales modificables como la hipertensión arterial, diabetes y dislipidemia.

A pesar de la evidencia precedente, lo que se aprecia en nuestro registro y en los realizados en otros países, es una disminución importante en el número de pacientes atendidos por IMCEST. El trabajo publicado por Rodríguez Leor et al. ${ }^{5}$ describe una reducción del $40 \%$ en el intervencionismo coronario percutáneo por IMCEST en 81 centros españoles que respondieron a una encuesta sobre la actividad asistencial en sus instituciones. La misma tendencia ha sido reportada por García et al. ${ }^{10}$ quienes describen una reducción de 38\% en las activaciones de los laboratorios de cateterismo cardíaco por IMCEST en 9 centros de alto volumen en los Estados Unidos. Reducciones menores se describen en Italia donde De Filippo et al. ${ }^{16}$ reportaron una caída de $22 \%$ en las admisiones por IMCEST en 15 hospitales de la región de Lombardía. Aunque los estudios descritos se han realizado en algunas de las zonas más afectadas por la pandemia del COVID-19 también se reproduce la tendencia en zonas de menor afectación como es el caso de Austria donde Metzler et al. ${ }^{17}$ encuentran una reducción de $39.4 \%$ en las admisiones por síndrome coronario agudo y $26 \%$ por IMCEST.

Se han planteado múltiples hipótesis respecto a las causas de la reducción del volumen de casos atendidos de
IMCEST. Una primera posibilidad a mencionar es el temor que tiene el paciente a acudir a un centro hospitalario donde podría contagiarse con la infección. Este aspecto ha sido evidenciado por la encuesta realizada por el Colegio Americano de Médicos de Emergencias donde el $80 \%$ de los encuestados manifiesta su temor al contagio al acudir a una sala de emergencia y $29 \%$ refiere que evitará cualquier consulta médica durante la pandemia. ${ }^{18}$ Al no acudir en busca de atención médica es posible que se presente un incremento de las complicaciones del IMCEST no reperfundido incluida la muerte súbita. Baldi et al $^{19}$ reportaron un aumento de $23 \%$ en la incidencia de arresto cardíaco extra-hospitalario no asociado a COVID-19 en 4 provincias de Lombardía, Italia. También se ha planteado un sub-diagnóstico como causa de la reducción de ingresos por IMCEST, ya que algunos síntomas del infarto de miocardio podrían solaparse con los del COVID-19 como reportan Yousefzai et al. 20

Nuestro registro muestra una tendencia a que los pacientes reciban menos terapias de reperfusión; con incremento en el uso de fibrinólisis y reducción de la angioplastia primaria lo que podría corresponder a cambios en protocolos de manejo y gestión de salas de hemodinámica durante la pandemia como ha sido reportado por Rodríguez-Leor et al. ${ }^{5}$ No hubo variación estadísticamente significativa en los tiempos evaluados (primer contacto médico, isquemia a reperfusión, puerta-balón), con una tendencia a la reducción del tiempo al primer contacto médico, a diferencia de la serie de casos reportada por Tam et al. ${ }^{9}$ en un hospital de Hong Kong 
donde se evidenció la prolongación de los tiempos mencionados. Este hallazgo podría explicarse por la reducción del tiempo de transporte de nuestros pacientes, al estar restringida la circulación vehicular durante el período de estado de emergencia salvo para emergencias médicas. Asimismo la reducción del tiempo de isquemia a reperfusión podría justificar la menor incidencia de falla cardíaca post infarto a pesar de la reducción en las terapias de reperfusión.

A pesar de no encontrar diferencias significativas en los tiempos promedio de atención ni en mortalidad hay un aspecto importante a destacar que es el incremento significativo de la proporción de pacientes con presentación tardía (>24 horas) como motivo de no reperfusión; es posible que el temor de contagio al acudir al hospital explique este hallazgo.

Como limitaciones de este subanálisis podemos señalar que si bien el registro PERSTEMI II del 2020 es de alcance nacional, los datos evaluados corresponden principalmente a centros pertenecientes al sistema público de salud ubicados en la ciudad de Lima y el Callao, por lo que sus conclusiones no necesariamente reflejan la situación de los ingresos por IMCEST en todo el territorio nacional. Se realizó un análisis retrospectivo, y no fue posible realizar la comparación con los meses correspondientes del año 2019, al no contar con dicha información.

\section{Conclusiones}

El presente subanálisis del registro PERSTEMI II muestra una reducción del 59\% en los ingresos por IMCEST durante los primeros 45 días del estado de emergencia por COVID-19 en el Perú respecto a los 45 días previos a éste. No hubo variación significativa en los tiempos de primer contacto médico, isquemia a reperfusión y puerta balón; sin embargo se evidenció tendencia a menor empleo de terapias de reperfusión y se incrementó la presentación tardía de los pacientes ( $>24$ horas) como causa de no reperfusión.

Esta reducción de las atenciones por IMCEST, podría tener como consecuencia el incremento de morbimortalidad, lo que obliga a las autoridades sanitarias a informar a la población en general sobre los síntomas y signos de alarma que deben motivar la consulta hospitalaria de emergencia.

\section{Agradecimientos}

A los investigadores del registro PERSTEMI II: Chacón M, Rodríguez R, Montesinos A, Gamio F, García A, Rotta A, Llerena N, Rojas P, Isla R, Barrientos C, Yabar G, Uribe J, Saavedra W, Aldazabal A.

\section{Referencias Bibliográficas}

1. Chan K, Zheng J, Mok $Y$ et al. SARS: prognosis, outcome and sequelae. Respirology. 2003;8(1):36-40. DOI: 10.1046/j.14401843.2003.00522.x

2. Bi $Q$, Wu $Y$, Ye $C$ et al. Epidemiology and transmission of COVID-19 in 391 cases and 1286 of their close contacts in Shenzhen, China: a retrospective cohort study. Lancet Infect Dis. 2020 Apr:1-6. DOI: 10.1101/2020.03.03.20028423

3. Wang D, Hu B, Hu C et al. Clinical Characteristics of 138 Hospitalized Patients With 2019 Novel Coronavirus-Infected Pneumonia in Wuhan, China. JAMA. 2020;323(11):1031-69. DOI: 10.1001/jama.2020.1585

4. Gobierno del Perú. Decreto de urgencia que establece diversas medidas excepcionales y temporales para prevenir la propagación del coronavirus (COVID-19) en el territorio nacional. [internet]. Lima: Dirección General de Gestión del Riesgo de Desastres y Defensa Nacional en Salud; 2020 [citado el 25 de Mayo de 2020]. Disponible en: https://busquedas.elperuano.pe/normaslegales/decreto-de-urgencia-que-establecediversas-medidas-excepcion-decreto-de-urgencia-n-0262020-1864948-1/

5. Rodriguez-Leor O, Cid-Álvarez B, Ojeda S et al. Impacto de la pandemia de COVID-19 sobre la actividad asistencial en cardiología intervencionista en España. REC Interv Cardiol. 2020;2:82-89. DOI: 10.24875/RECIC.M20000120

6. Nakashima T, Tahara Y. Achieving the earliest possible reperfusion in patients with acute coronary syndrome: a current overview. J Intensive Care. 2018;6(20):1-10. DOI: 10.1186/ s40560-018-0285-9

7. Chacón-Diaz M, Vega A, Aráoz O et al. Características epidemiológicas del infarto de miocardio con elevación del segmento ST en Perú: resultados del PEruvian Registry of ST-segment Elevation Myocardial Infarction (PERSTEMI). Arch Cardiol Mex. 2018;88(5):403-12. DOI: 10.1016/j.acmx.2017.11.009

8. Ibañez B, James S, Agewall S et al. Guía ESC 2017 sobre el tratamiento del infarto agudo de miocardio en pacientes con elevación del segmento ST. Rev Esp Cardiol. 2017;70(12):1-61. DOI: 10.1016/j.recesp.2017.10.048

9. Tam C, Cheung K, Lam S et al. Impact of Coronavirus Disease 2019 (COVID-19) Outbreak on ST-Segment-Elevation Myocardial Infarction Care in Hong Kong, China. Circ Cardiovasc Qual Outcomes. 2020;13(4):1-3. DOI: 10.1161/CIRCOUTCOMES.120. 006631

10. Garcia S, Albaghdadi M, Meraj P et al. Reduction in ST-Segment Elevation Cardiac Catheterization Laboratory Activations in the United States during COVID-19 Pandemic. J Am Coll Cardiol. 2020;75(22):2871-72. DOI: 10.1016/j.jacc.2020.04.011

11. Kwong J, Schwartz K, Campitelli M. Acute Myocardial Infarction after Laboratory-Confirmed Influenza Infection. N Eng J Med. 2018;378(4):345-53. DOI: 10.1056/NEJMc1805679

12. Tsui $K$, Leung $T$, Yam L et al T. Coronary plague instability in severe acute respiratory syndrome. Int J Cardiol. 2005;99(3): 471-72. DOI: 10.1016/j.ijcard.2003.11.052

13. Peiris J, Chu C, Cheng V et al. Clinical progression and viral load in a community outbreak of coronavirus-associated SARS pneumonia: a prospective study. Lancet Infect Dis. 2003; 361 (9371): 1767-72. DOI: 10.1016/S0140-6736(03)13412-5 
14. Mittleman M, Mostofsky E. Physical, Psychological and Chemical Triggers of Acute Cardiovascular Events. Preventive Strategies. Circulation. 2011;124(3):346-54. DOI: 10.1161\%2FCIRCULATIONAHA.110.968776

15. Dimsdale J. Psychological Stress and Cardiovascular Disease. J Am Coll Cardiol. 2008; 51(13):1237-46. DOI: 10.1016\%2Fj.jacc. 2007.12.024

16. De Filippo O, D’Ascenzo F, Angelini F et al. Reduced Rate of Hospital Admissions for ACS during Covid-19 Outbreak in Northern Italy. N Eng J Med. 2020;383:88-89. DOI: 10.1056/ NEJMc2009166

17. Metzler B, Siostrzonek P, Binder R et al. Decline of acute coronary sindrome admissions in Austria since the outbreak of COVID-19: the pandemic response causes cardiac collateral damage. Eur Heart J. 2020;41(19):52-53. DOI: 10.1093/eurheartj/ehaa314

18. American College of Emergency Physicians. Public Poll: Emergency Care Concerns amidst COVID-19. US: ACEP. 2020. Disponible en: https://www.emergencyphysicians.org/article/ covid19/public-poll-emergency-care-concerns-amidst-covid19

19. Baldi E, Sechi G, Mare C et al. Out-of-Hospital cardiac Arrest during the Covid-19 Outbreak in Italy. N Eng J Med. 2020. DOI: 10.1056/NEJMc2010418

20. Yousefzai R, Bhimaraj A. Misdiagnosis in the COVID era: When Zebras are Everywhere, Don't Forget the Horses. JACC Case Rep. 2020. DOI: 10.1016/j.jaccas.2020.04.018 